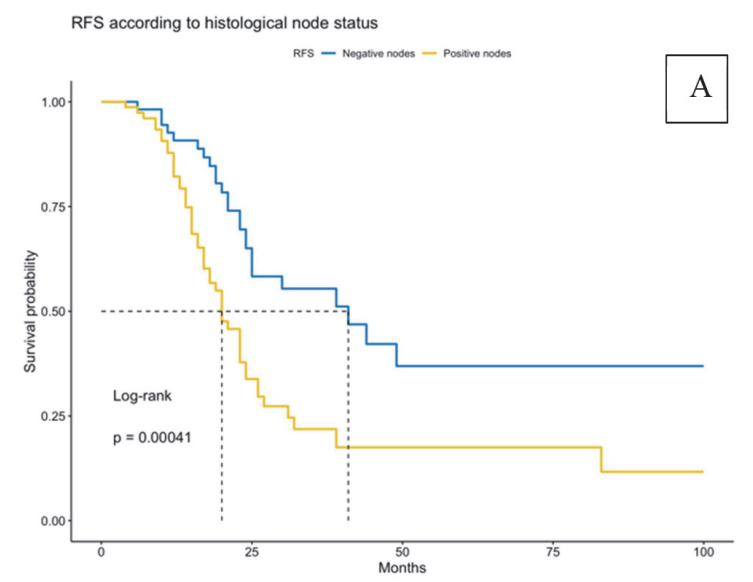

OS according to histological node status

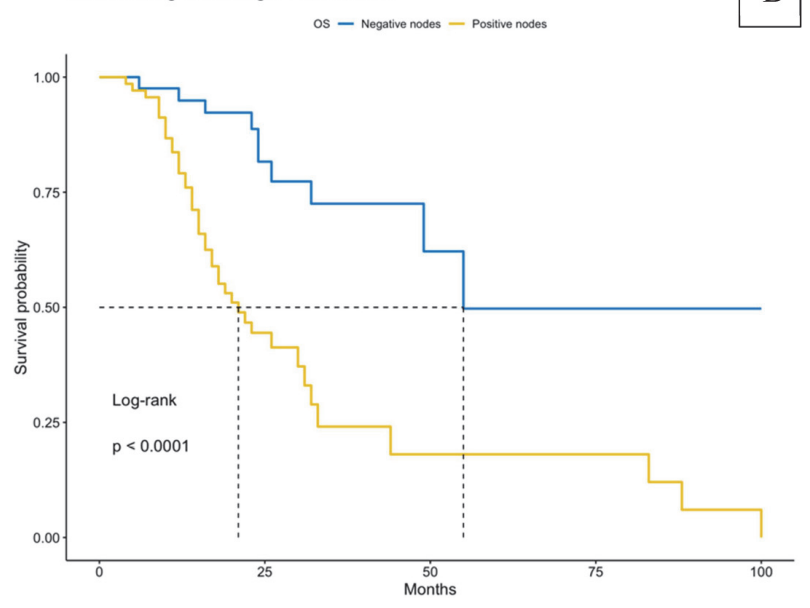

Abstract 240 Figure 2 Patients with a stage IIB-IV high-grade serous ovarian cancer who underwent neoadjuvant chemotherapy followed by interval debulking surgery with a systematic lymphadenectomy. A) Recurrence free survival (RFS) according to node status after neoadjuvant chemotherapy $(p<0.001)$; $B)$ Overall survival (OS) according to node status after neo-adjuvant chemotherapy $(p<0.001)$

lymphadenectomy and no lymphadenectomy group concerning 2-year RFS (47.4\% and 48.6\%, p=0.87, respectively) and 5year OS $(63.2 \%$ versus $58.6 \%, \mathrm{p}=0.41$, respectively). Postoperative complications tended to be more frequent in the group with a lymphadenectomy (18.57 versus $31.58, \mathrm{p}=0.09$ ). In the group of patients with lymphadenectomy, survival was significantly altered in patients with involved nodes (positive lymph nodes: 2-year RFS $42.5 \%$ and 5-year OS 49.4\%, negative nodes: 2-year RFS $60.7 \%$ and 5-year OS $82.2 \%, \mathrm{p}=$ 0.03 and $\mathrm{p}<0.001$, respectively).

Conclusion* Lymphadenectomy during IDS does not improve survival and increases post-operative complications.

\section{IMPACT OF CO-MEDICATION ON SURVIVAL IN PATIENTS WITH OVARIAN CANCER - A ANALYSIS OF 4 PROSPECTIVE TRIALS OF THE AGO-OVAR AND ENGOT/ GCIG COLLABORATORS}

${ }^{1} \mathrm{D}$ Denschlag*, ${ }^{2} \mathrm{~F}$ Heitz, ${ }^{3} \mathrm{~J}$ Pfisterer, ${ }^{4} \mathrm{D}$ Tutschkow, ${ }^{5} \mathrm{~W}$ Meier, ${ }^{2} \mathrm{P}$ Harter, ${ }^{6} \mathrm{P}$ Wimberger, ${ }^{7}$ MR Mirza, ${ }^{8}$ Ray-Coquard, ${ }^{9} \mathrm{G}$ Scambia, ${ }^{10} \mathrm{JW}$ Kim, ${ }^{11} \mathrm{~N}$ Colombo, ${ }^{12} \mathrm{~A}$ Oaknin, ${ }^{13} \mathrm{~J}$ Sehouli, ${ }^{14} \mathrm{~K}$ Lindemann, ${ }^{15} \mathrm{~A}$ Floquet, ${ }^{16} \mathrm{M}$ Eichbaum, ${ }^{1} \mathrm{~S}$ Spiegelberg, ${ }^{13} \mathrm{H}$ Woopen, ${ }^{2} \mathrm{~A}$ Du Bois. ${ }^{1}$ Hochtaunus-Klinken Bad Homburg, Gynecology, Bad Homburg, Germany; ${ }^{2}$ Klinikum Essen Mitte, Gynecologic Oncology; ${ }^{3}$ Gynecologic Oncology Center Kiel; ${ }^{4}$ University of Marburg, Center for Clinical Trials, Germany; ${ }^{5}$ University of Düsseldorf, Germany; ${ }^{6}$ University of Dresden, Gynecology, Germany; ${ }^{7}$ Copenhagen University Hospital, Department of Oncology, Copenhagen, Denmark; ${ }^{8}$ Centre Leon Berard, Universite Claude Bernard Lyon Est, France; ${ }^{9}$ Universita Cattolica del Sacro Cuore, Policlinico Gemelli, Rome, Italy; ${ }^{10}$ Seoul National University, Obstetrics and Geynecology, Seoul, Korea, Dem. People's Rep. Of; ${ }^{11}$ European Institute of Oncology, Gynecologic Oncology, Milano, Italy; ${ }^{12}$ Vall d'Hebron University Hospital, Barcelona, Spain; ${ }^{13}$ Charite University Hospital Campus Virchow, Gynecologic Oncology, Germany; ${ }^{14}$ Oslo University Hospital Division of Cancer Medicine, Gynecologic Oncology, Oslo, Norway; ${ }^{15}$ Institut Bergonie, Bordeaux Cedex, France; ${ }^{16}$ Helios Dr. Horst Schmidt Kliniken, Gynecology, Wiesbaden, Germany

\subsection{6/ijgc-2021-ESGO.366}

Introduction/Background* There is poor evidence from mostly retrospective series whether co-medication with metformin, statin or beta-blocker have an impact on survival in patients with primary ovarian cancer. The aim of this study was to investigate the association of these medications with survival. Methodology Individual data from 3 prospective phase III randomized controlled trials (AGO-OVAR 11/ICON 7, 12, 16) and one phase II trial (AGO-OVAR 15) were pooled and analyzed. Patients were either classified as "ever-user" if the specific co-medication was documented at least once during the trial. In. contrast, "never-users" served as controls.

Data were adjusted for potential confounders (age, FIGO stage, histology, residual tumor after surgery, ECOG performance status, BMI, Charlson Comorbidity Index and assigned treatment within the trial) in multivariate Cox regression analyses.

Result(s)* Overall, 2.857 patients were included in the analyses. "Ever-users" were: $\mathrm{N}=100$ patients received metformin (3.5\%), $\mathrm{N}=226$ received statins (7.9\%), and $\mathrm{N}=475$ (16.6\%) received beta-blockers (BB) $(\mathrm{N}=391$ selective (sBB); $N=84$ non-selective (nsBB)) as co-medication.

There were no significant differences regarding the baseline characteristics (histology, FIGO stage, residual tumor after surgery, and chemotherapy-schedule) between "ever- and neverusers" except that "ever-users" were significantly older and more obese, compared to controls.

Median progression-free (PFS) and overall survival (OS) for the entire cohort was 18.7 months and 60.1 months, respectively

Multivariate analyses for PFS and OS including age, BMI, Histology, FIGO stage, residual tumor after surgery, ECOG performance status and CCI revealed neither a significant impact of metformin on survival of "ever-users", compared to "never-users" (PFS HR 0.94 95\%-CI 0.69-1.29, p=0.7; OS HR 0.82 95\%-CI $0.58-1.17, \mathrm{p}=0.28$ ), nor for statins (PFS HR 0.98 95\%-CI $0.82-1.18, \mathrm{p}=0.87$; OS HR $0.9195 \%$-CI 
0.74-1.12, $\mathrm{p}=0.37$ ), respectively. In contrast, "ever-users" of sBB had a significantly elevated risk for recurrence and death in multivariate analysis (PFS HR 1.22 95\%-CI 1.05-1.41, $\mathrm{p}=0.009$; OS HR 1.25 95\%-CI 1.06-1.47, $\mathrm{p}=0.009$ ).

Conclusion* In this large pooled analysis neither a co-medication with metformin nor with statins had a significant impact on survival in patients with primary ovarian cancer. In contrast, co-medication with a beta-blocker was associated with worse survival. Further studies are warranted to confirm this observation.

\section{PARP-INHIBITORS BEYOND PROGRESSION: A NEW WAY TO MANAGE OLIGOMETASTATIC OVARIAN CANCER RECURRENCE}

${ }^{1} \mathrm{C}$ Marchetti ${ }^{*},{ }^{1} \mathrm{E}$ Palluzzi, ${ }^{1} \mathrm{~S}$ Cappuccio, ${ }^{1} \mathrm{G}$ Avesani, ${ }^{1} \mathrm{~A}$ Nardangeli, ${ }^{1,2} \mathrm{G}$ Scambia, ${ }^{1,2}$ A Fagotti. ${ }^{1}$ Fondazione Policlinico Agostino Gemelli-IRCCS, Dipartimento di Scienze della Salute della Donna, del Bambino e di Sanità Pubblica, Rome, Italy; ${ }^{2}$ Università Cattolica del Sacro Cuore, Rome, Italy

\subsection{6/ijgc-2021-ESG0.367}

Introduction/Background* The benefit of surgery and maintenance treatment with parp inhibitors (PARPi) has been recently shown in ovarian cancer (OC) recurrence. Also, the efficacy and safety of stereotactic body radiotherapy (SBRT) is demonstrated in patients with metastatic, persistent, and recurrent OC. The management of oligometastatic progression (OMP) during PARPi maintenance is unclear and continuing the treatment beyond progression could be an option.

Methodology This is an observational, retrospective, single arm study. Patients affected by OC recurrence treated with PARPi

\begin{tabular}{ll} 
Abstract 242 Table 1 & \\
\hline & PARPi manteinance \\
\hline & Nr. (\%) \\
All patients & 24 \\
Median age (years) (range) & $51(35-67)$ \\
Histology & \\
Serous & $24(100)$ \\
PARPi & \\
Olaparib & $9(38)$ \\
Niraparib & $15(62)$ \\
BRCA status & \\
BRCA MT & $10(42)$ \\
BRCA WT & $14(58)$ \\
Therapy at oligometastatic progression & \\
Surgery & $9(38)$ \\
SBRT & $15(62)$ \\
Site of oligometastatic progression & \\
Lynphnode & $14(58)$ \\
Peritoneal & $4(17)$ \\
Parenchymal disease & $6(25)$ \\
PFS & Months \\
Median PFS1 & $(\mathrm{Cl})$ \\
& 23 months \\
Median ppPFS & $(\mathrm{Cl} 95 \% 11-34)$ \\
Median bpPFS & 6 months \\
& $(\mathrm{Cl} 95 \% 5-7)$ \\
\hline & 29 months \\
(CI 95\% 17 - 40) \\
\hline
\end{tabular}
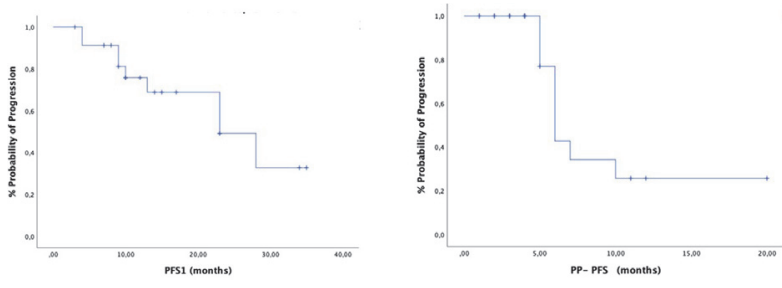

Abstract 242 Figure 1

in maintenance setting received surgery or SBRT, if OMP occurred. OMP was assessed by either CT scan or PET/CT scan, in case of isolated disease progression (one nodule) or discrete diffusion (up to three nodules in different locations) or progression in "sanctuary" site. Maintenance treatment was continued until extensive progression of disease. Primary objectives were: Progression Free Survival 1 (PFS1), defined as the time elapsed from the start of PARPi and OMP; post-progression-PFS (ppPFS), defined as the time elapsed from OMP and the last follow up (FU). Beyond-progression PFS (bpPFS), defined as the time elapsed from the start of PARPi and the definitive progression of disease or last FU (PFS1+ppPFS), and efficacy of surgery versus SBRT at OMP were secondary objectives.

Result(s)* From June 2017 to December 2020186 OC patients were treated with PARPi maintenance at recurrence. Of these $24(13 \%)$ developed OMP (58\% lymphnodes, 17\% peritoneal, $25 \%$ visceral disease). Median age was 51 years. Olaparib and Niraparib maintenance were administered to 9 (38\%) and 15 (62\%) patients, respectively. Median PFS1 was 23 months [Confidence Interval (CI) 95\% 11 - 34]. When OMP occurred $9(38 \%)$ and $15(62 \%)$ pts were subjected to surgery and SBRT, respectively. Median ppPFS was 6 months (CI 95\% 5 - 7). At the time of this publication $62.5 \%$ patients are still on treatment with PARPi beyond progression. Conclusion* OC patients, who have an OMP during PARPi maintenance at recurrence, may continue to benefit from PARPi treatment if combined with local treatment. Molecular assessment at oligometastatic and extensive progression could provide further information to define PARPi resistance mechanisms according to the type of disease progression.

\section{PFS OF ELDERLY OVARIAN CANCER PATIENTS MIGHT BE PREDICTED BY G-8 GERIATRIC SCREENING TOOL - RESULTS OF A RETROSPECTIVE COHORT STUDY}

${ }^{1} \mathrm{~K}$ Anic*, ${ }^{1} S$ Birkert, ${ }^{1} \mathrm{R}$ Schwab, 'MW Schmidt, ${ }^{1} \mathrm{~V}$ Linz, ${ }^{1} S$ Krajnak, ${ }^{1} \mathrm{~A}-\mathrm{S}$ Heimes, ${ }^{1} \mathrm{M}$ Schmidt, ${ }^{2} \mathrm{C}$ Westphalen, ${ }^{3}$ EK Hartmann, ${ }^{1} \mathrm{~A}$ Hasenburg, ${ }^{1} \mathrm{M}$ Battista. ${ }^{1}$ University Medical Centre of the Johannes Gutenberg University Mainz, Department of Gynaecology and Obstetrics, Mainz, GERMANY; ${ }^{2}$ University Medical Centre of the Johannes Gutenberg University Mainz, Department of Geriatric Medicine, Mainz, GERMANY; ${ }^{3}$ University Medical Centre of the Johannes Gutenberg University Mainz, Department of Anesthesiology, Mainz, GERMANY

\subsection{6/ijgc-2021-ESG0.368}

Introduction/Background* The aim of this study was to evaluate the impact of the preoperative global health status on the prognosis of patients with ovarian cancer (OC) older than 60 years, who received cytoreductive surgery.

Methodology G-8 geriatric screening tool (G-8 score), Lee Schonberg prognostic index, Eastern Cooperative Oncology Group (ECOG) performance status and Charlson Comorbidity Index (CCI) were determined retrospectively in a consecutive 